\title{
Drug-resistant epilepsy: modern concepts, integrative mechanisms, and therapeutic advances
}

\author{
${ }^{1,2 *}$ Vitalie Chiosa, ${ }^{2,3,4}$ Dumitru Ciolac, ${ }^{2,5}$ Viorica Chelban, ${ }^{1,2,3}$ Daniela Gasnas, \\ ${ }^{1,2}$ Anatolie Vataman, ${ }^{2,3}$ Cristina Munteanu, ${ }^{1,2,3,4}$ Stanislav Groppa \\ Contributing authors: ${ }^{1,2,3}$ Pavel Leahu, ${ }^{2,4}$ Elena Condratiuc, ${ }^{1,2,4}$ Daniela Aftene, ${ }^{2,4}$ Diana Dragan, ${ }^{2,3}$ Renata Racila, \\ ${ }^{2,4}$ Natalia Doten, ${ }^{1,2,4}$ Natalia Stoianov, ${ }^{1,2,3}$ Alexandra Condrea, ${ }^{1,2,3}$ Doina Ropot, ${ }^{1,2,3}$ Maria Vasilieva \\ ${ }^{1}$ Department of Neurology No 2, ${ }^{2}$ Laboratory of Neurobiology and Medical Genetics, \\ Nicolae Testemitanu State University of Medicine and Pharmacy, Chisinau, the Republic of Moldova \\ ${ }^{3}$ Department of Neurology, Epileptology and Internal Diseases, ${ }^{4}$ National Center for Epileptology \\ Institute of Emergency Medicine, Chisinau, the Republic of Moldova \\ ${ }^{5}$ Department of Neuromuscular Diseases, Queen Square Institute of Neurology, University College London
}

Authors' ORCID iDs, academic degrees, and contributions are available at the end of the article

*Corresponding author - Vitalie Chiosa, e-mail: vitalie.chiosa@usmf.md

Manuscript received September 17, 2021; revised manuscript October 01, 2021; published online October 12, 2021

\begin{abstract}
Background: Drug-resistant epilepsy is the cause of severe disability. Multiple questions remain unanswered both in terms of pathogenesis and therapeutic management. For this narrative review, PubMed database and Infomedica library were searched by using "drug-resistance in epilepsy" and "treatment of drug-resistant epilepsy" as key words. The following filters were applied: "Clinical Trial", "Meta-analysis", "Multicenter Study", and "Randomized Controlled Trial", covering the period of 01.01.2005-06.01.2021.Several hypotheses have been proposed, i.e., pharmacokinetic, intrinsic severity, gene, target, transporter, and neural network hypotheses. Many controlled trials showed different results in terms of seizure control after combined methods of therapies. Immunotherapy, palliative epilepsy surgery alone or associated with neurostimulation procedures including vagus nerve, trigeminal nerve, or deep brain stimulation may be efficient, however, seizure freedom is not always achieved. Genetic epilepsies might benefit from gene and exosome therapy; however, further studies are needed to verify their safety.

Conclusions: Neuroscience of drug-resistant epilepsy faces many challenges. Inflammatory mediators, biomarkers, and genes might allow the identification of new treatment targets, contribute to an earlier diagnosis, and assess the clinical outcomes.

Key words: drug-resistant epilepsy, hypotheses of drug-resistance, therapeutic advances.
\end{abstract}

Cite this article

Chiosa V, Ciolac D, Chelban V, Gasnas D, Vataman A, Munteanu C, Groppa S. Drug-resistant epilepsy: modern concepts, integrative mechanisms, and therapeutic advances. Mold Med J. 2021;64(4):72-85. https://doi.org/10.52418/moldovan-med-j.64-4.21.14.

\section{Introduction}

Epilepsy is a relatively common neurological disease defined by two unprovoked seizures (or one unprovoked seizure with the likelihood of more) that were not caused by some known and reversible medical condition [1]. It affects about $0.5-1 \%$ of the population worldwide or absolutely about 50 million of population, around half of them (24 millions) diagnosed with active idiopathic epilepsy [2]. Approximately $10 \%$ of world population will experience a seizure at some point during their lifetime [3].The incidence of epilepsy is about 61 per 100000 persons-years and was higher in low/middle-income countries than in highincome countries, 139.0 vs 48.9 [3]. Epilepsy is still an important cause of disability ( $25 \%$ of people with epilepsy have intellectual disability [4]), mortality, and is surrounded by prejudice and stigma - a well-documented barrier to health seeking behavior, engagement in care and adherence to treatment across a range of health conditions globally [5], perceived by cohabiting relatives of people with epilepsy and surrounded people. Higher perceived stigma is significantly related to generalized seizures, longer disease duration [6], and higher perceptions of stigma are associated with worse quality of life in people with epilepsy. SUDEP is a life-threatening condition with an estimated incidence of 0.58 to 9.0 per 1000 persons-years with a $35 \%$ of lifetime cumulative risk in refractory epilepsy patients [7] that is playing an important role in the quality of life of these patients. People with epilepsy have a higher risk of sudden death, with reported annual incidences of 1 per 1000. For those with uncontrolled epilepsy, the incidence is higher as 1 per 200 [8], but the lowest incidence is in children and consists $0-0.2$ per 1000 [9]. The risk of sudden death is greater in women, representing 1.45 per 1000 than in men 0.98 per 1000 [10]. The epilepsy treatment with antiepileptic drugs has limited ef- 
fectiveness. In case of correct diagnosis, $70 \%$ of patients with epilepsy are drug sensitive and seizure free, $50 \%$ of them after initial monotherapy [11], 10\% in context of alternative monotherapy and $10 \%$ with first add-on or polytherapy. The remaining $30 \%$ are drug resistant. These data may fluctuate with $25 \%$ range of drug-resistance [12].

\section{The modern definition of drug resistance}

The concept drug-resistant epilepsy means pharmacoresistant, drug resistant, refractory or medically intractable epilepsy [13]. The definition evaluated during the last years as: a) seizures which have not been completely controlled with AEDs 1 year after onset despite accurate diagnosis and carefully monitored treatment [14]; b) seizures of sufficient frequency and severity after 2 years of AED treatment [15]; c) the patient is not seizure free after 1 year treatment with 2-3 AED [16]. The ILAE consensus concerning definition was obtained in 2009 and it is: failure of adequate trials of two tolerated and appropriately chosen and used AEDs (whether as monotherapies or in combination) to achieve sustained seizure freedom [13]. Discussing about pharmacoresistant epilepsy is very important to rule out cases of nonadherence in epilepsy and cases of pseudorefractory epilepsy [17] The approach of patient with pharmacoresistant epilepsy is difficult and the first step would be the diagnosis reconsideration. The surgery is the superior option in treatment of these patients, but among the $30 \%$ of patients with confirmed diagnosis of pharmacoresistant epilepsy only 10-15\% become candidates for epilepsy surgery [18].

\section{Mechanisms of drug resistance}

Nowadays understanding the multifactorial mechanisms underlying drug-resistant epilepsy has the potential to contribute to more effective development of treatment options for patients with epilepsy. The combination of multiple mechanisms expressed in each individual patient represents the most popular hypothesis. A key limitation of the entire research is the difficulty of demonstrating whether the changes associated with drug-resistant epilepsy are an epiphenomenon of epileptogenesis [19].

Intrinsic severity hypothesis. Rogawski and Johnson proposed the hypothesis that the resistance of antiepileptic drugs is not due to specific drug resistance factors, but due to the degree of severity of epilepsy that is directly related to the response to treatment. This "intrinsic severity hypothesis" was later updated by Rogawski, who postulates that drug resistance is an inherent property of epilepsy that is directly related to the severity of the disease. The increased frequency or density of seizures that precede the onset of antiepileptic therapy is the most important factor associated with a reduced chance of long-term remission of the disease [20]. Although the high frequency of seizures is a predictor of drug resistance, but it is clearly not the only one. The intrinsic severity hypothesis says that common neurobiological factors contribute to both the severity of epilepsy and pharmaceutical co-resistance. Other features of the severity of the pathological condition, such as the extent of structural damage or behavioral phenotype, are also predictors of resistance to antiepileptic drugs. Although the intrinsic severity hypothesis seems biologically possible, it was not applied to disease with a fluctuating or evolving pattern of resistance. In addition, there is very limited evidence to support a direct link between the severity of epilepsy and response to treatment [21]. In this regard, data from studies supporting the hypothesis of intrinsic severity suggest that the high frequency of pre-treatment seizures is an important predisposing factor for refractory epilepsy [22]. A similar conclusion was drawn from a study on children diagnosed with epilepsy, which showed that administration of anticonvulsant therapy during the period of first ten seizures had no aggravating or early remission effect [23]. In another randomized study of 1847 patients with epilepsy, the authors compared the effectiveness of immediate treatment with that of the delayed treatment and found that immediate treatment was associated with reduced seizure frequency in the first 1-2 years, but long-term remission rates did have no difference between the two groups. Therefore, it has been suggested that the theory of intrinsic severity does not sufficiently explain the mechanism of drug resistance in epilepsy [21].

Target hypothesis. Among the various mechanisms of drug resistance that have been proposed, the target hypothesis postulates that acquired (epilepsy-induced) alterations to the structure and/or functionality of brain targets of antiseizure drugs (ASDs) lead to a reduction in their sensitivity to treatment and thus lead to refractoriness [24]. To exhibit antiseizure activity, a drug must act on one or more target molecules in the brain, and these targets include voltagedependent ion channels, neurotransmitter receptors, and transporters or metabolic enzymes involved in the release, uptake, and metabolism of neurotransmitters [25].

The target hypothesis is primarily based on studies with carbamazepine on voltage-gated sodium channels in hippocampal neurons (CA1 and dentate granule cells). Remy et al. [26] showed that the use dependent block of voltage-dependent $\mathrm{Na}+$ channels of dentate granule cells by carbamazepine is completely lost in patients with carbamazepine-resistant temporal lobe epilepsy (TLE), and the fast recovery from inactivation of the fast $\mathrm{Na}+$ current was carbamazepineinsensitive in pharmacoresistant patients [27]. Also, the authors suggested that a loss of $\mathrm{Na}+$ channel drug sensitivity could explain the development of drug-resistant epilepsy (DRE). In another study, Remy et al. [28] demonstrated that the effects of phenytoin on fast recovery from inactivation of $\mathrm{Na}+$ channels of hippocampal granule neurons were significantly reduced, though not as pronounced as observed with carbamazepine, and that lamotrigine slowed the time course of recovery from fast inactivation. Thus, these results suggested that target mechanisms of drug resistance are cell type- and ASD-specific. Newly, Doeser et al. [29] reported that eslicarbazepine may possess advantages over conventional $\mathrm{Na}+$ channel modulators, because it maintained activity in chronically epileptic tissue. One possibility for altered 
sensitivity of $\mathrm{Na}+$ channels in CA1 or dentate granule cells in epileptic tissue is that the subunit composition of these channels is altered, resulting in channels with lower ASD sensitivity [24].

Other drug targets, such as $\mathrm{GABA}_{\mathrm{A}}$ receptors, may be altered in patients and animal models with intractable epilepsy [27]. Profound alterations in $\mathrm{GABA}_{\mathrm{A}}$ receptor subtype expression have also been reported in adult patients with ASD-resistant TLE and pediatric epilepsy patients undergoing epilepsy surgery. Brooks-Kayal et al. [30], using the rat pilocarpine model of TLE, demonstrated that expression of $\mathrm{GABA}_{\mathrm{A}}$ receptor subunit mRNAs is substantially altered in hippocampal dentate granule cells of pilocarpine treated rats versus controls. These changes in $\mathrm{GABA}_{\mathrm{A}}$ receptor subunit expression correlated with profound alterations in receptor function and pharmacology. In addition to the enhanced zinc sensitivity, $\mathrm{GABA}_{\mathrm{A}}$ receptors from the epileptic hippocampus lose their sensitivity to augmentation by the benzodiazepine site modulator zolpidem. However, none of these studies examined whether ASD-resistant epileptic rats differ from responsive rats in these changes in $\mathrm{GABA}_{\mathrm{A}}$ receptor function [27].

Although the target hypothesis is a biologically plausible theory to explain drug resistance, the fact that most drugresistant patients are resistant to several ASDs acting on different therapeutic targets undermines the general utility of the target hypothesis and instead supports the existence of a mechanism nonspecific to individual ASDs [31].

Transporter hypothesis. The transporter hypothesis is based on two assumptions: (1) overexpression of efflux transporters correlates with pharmacoresistance in epilepsy and (2) antiepileptic drugs (AEDs) are subject to active transport by efflux transporters [31].

Overexpression of efflux transporters at the blood-brain barrier is discussed as one factor that might limit brain penetration and efficacy of AEDs. The best understood efflux transporters are members of the ABC (ATP-binding cassette) superfamily subfamilies B, C, and G, specifically P-glycoprotein (ABCB1 or MDR1), the multidrug resistanceassociated proteins (MRP1, ABCC1; MRP2, ABCC2), and breast cancer resistance protein (BCRP, ABCG2) [31].

P-glycoprotein (P-gp), also known as MDR1 or ATPbinding cassette subfamily B member 1 (ABCB1), actively exports hydrophobic and amphipathic molecules from the inside of cells or membranes to the outside, as a critical defense mechanism. Its overexpression in epileptogenic brain tissue in patients with refractory epilepsy has been documented in numerous studies. For the first time overexpression of MDR1 mRNA was demonstrated in 11 out of 19 resected brain specimens from patients with refractory focal epilepsy. P-gp overexpression was also detected in astrocytes and/or dysplastic neurons in common pathological causes of refractory epilepsy, including dysembryoplastic neuroepithelial tumors (DNT), hippocampal sclerosis (HS), and focal cortical dysplasia (FCD) [32].
The overexpression of a multidrug resistance-associated protein (MRP1) in astrocytes and/or dysplastic neurons in HS, DNT, and FCD has also been described [32]. The results confirm that MRP1 protein expression levels in astrocytes and neurons from brain tissue of epilepsy patients are significantly increased compared to brain tissue from healthy individuals, while endothelial MRP1 expression did not differ between the two [33]. It was reported increased MRP2 and MRP5 mRNA levels in endothelial cells isolated from epileptic brain tissue of patients with refractory epilepsy compared to control endothelial cells from human umbilical vein and aneurysm domes. Aronica et al. [34] reported MRP2 protein overexpression in endothelial cells and astrocytes in HS tissue specimens of adult patients with temporal lobe epilepsy (TLE).

Similar to P-gp, BCRP (breast cancer resistance protein) transports a wide variety of substrates, and its tissue distribution contributes to its important roles in restricting absorption and facilitating elimination of drugs and xenobiotics, but due to the lack of evidence on BCRP overexpression in human epileptic brain tissue, BCRP is unlikely a major player in AED resistance as proposed by the transporter hypothesis [31].

Although increased mRNA and protein expression levels of P-gp and MRPs have been demonstrated in resected brain tissue from patients with AED-resistant epilepsy, previous studies did not include proper controls, as it is generally difficult to obtain brain tissue from either patient with drug-responsive epilepsy or from healthy subjects without brain disease. Therefore, it is still unclear if overexpression of efflux transporters correlates with and potentially causes AED resistance, or if it is an epiphenomenon of epilepsy in humans that is unrelated to AED resistance [35].

Conclusive evidence that AEDs are transported by efflux transporters at therapeutic concentrations is considered the weak link in the transporter hypothesis. Early studies suggested that several AEDs may be substrates for P-gp and/or MRPs. Researchers who attempted to identify AEDs as substrates of P-gp, MRPs, and/or BCRP mainly used three approaches: transporter-overexpressing cell lines, transporter inhibition in cell lines and/or in animals, and transporter gene knockout mice [36]. Each of these approaches has its own strengths and weaknesses. For example, transporter-overexpressing cell lines only allow in vitro analysis. Transporter inhibitors may lack specificity and interact with more than one transporter, and knockout mice may show potential compensatory upregulation of other transporters, which may complicate the situation. Therefore, all three approaches may need to be used together in one thorough study to obtain conclusive data [32]. In addition, compared to chemotherapeutic drugs that are usually high-affinity substrates for P-gp and MRPs, AEDs are weak substrates for the efflux transporters and more easily cross the blood-brain barrier under physiological conditions [37].

AED Transport by P-gp. P-glycoprotein transports primarily hydrophobic and amphipathic compounds [7]. 
Most AEDs are planar lipophilic molecules, and therefore, theoretically many AEDs should be P-gp substrates [19]. The first report of P-gp-mediated transport of an AED came from Tishler et al. [38], who reported lower steady-state intracellular phenytoin concentrations in MDR1-expressing neuroectodermal cells as compared to MDR1-negative cells. Phenobarbital, lamotrigine, felbamate, and oxcarbazepine were shown to be transported by $\mathrm{P}$-gp in rat brain microdialysis studies using verapamil as a P-gp inhibitor [39]. Owen et al. [40] concluded that carbamazepine was not a substrate for P-gp. In contrast, other studies, supported that P-gp transports carbamazepine. Data from another microdialysis study in rat suggest that P-gp does not transport levetiracetam and valproic acid [41].

Luna-Tortós et al. [42] pointed out that conventional bidirectional transport assays may not be suitable to identify AEDs as P-gp substrates due to the highly permeable nature of most AEDs. Using a modified transport assay (concentration equilibrium transport assay; CETA) which allows evaluating active transport separately from passive permeability, it was detected P-gp transport of phenytoin, phenobarbital, lamotrigine, levetiracetam, and topiramate, but not carbamazepine in MDR1-transfected LLC-PK1 cells. Zhang et al. [36] used both the cell monolayer bidirectional assay and CETA in MDR1-transfected MDCKII and LLC-PK1 cells to test if phenytoin, phenobarbital, or ethosuximide were transported by P-gp. Results from the CETA experiments suggested concentration-dependent P-gp transport of phenytoin in both MDCKII-MDR1 and LLC-PK1-MDR1 cells and transport of phenobarbital only in MDCKII-MDR1 cells.

The only clinical evidence linking overexpression of blood-brain barrier P-gp to reduced AED brain levels came from a pilot study by Marchi et al. [43], who demonstrated an inverse correlation between the brain-plasma concentration ratio of the major active metabolite of oxcarbazepine, 10,11-dihydro-10-hydroxy-5H-dibenzo(b,f)azepine-5carboxamide(10,11-dihydro-10-hydroxycarbama-zepine), and the MDR1 mRNA brain expression levels in resected epileptic tissue from patients with refractory epilepsy.

Since different models yield different results, both in vivo and in vitro data seem to be needed to identify which AEDs are substrates for which transporter [31]. In this regard, by combining the available evidence, Zhang et al. [35] suggested that lamotrigine, oxcarbazepine, phenobarbital, and phenytoin are considered definite P-gp substrates, because P-gp-mediated transport of these AEDs has been supported by both in vivo and in vitro evidence.

AED Transport by MRPs. MRPs transport neutral organic drugs and amphiphilic organic anions including drugs conjugated to glutathione, sulfate, glucuronate, and phosphate. Thus, it is possible that MRPs transport a number of AEDs and/or their metabolites and limit their access to the brain. Phenytoin transport by MRP1 and/or MRP2 was shown in vivo in normal rats using brain microdialysis with the MRP1/2 inhibitor probenecid. Carbamazepine and oxcarbazepine were shown to be substrates of MRP1 and/ or MRP2 in microdialysis in vivo studies with probenecid [39]. Valproic acid was the first AED found to be a substrate for MRPs in brain endothelial cells. Potschka et al. [44] showed that levetiracetam was not transported by MRP1/2. Baltes et al. [45] conducted bidirectional transport assays in monolayers of MRP2-transfected MDCKII kidney cells, and none of the AEDs tested (phenytoin, levetiracetam, carbamazepine) was found to be transported by MRP2.

Using CETA in MDCKII kidney cells transfected with human MRP1, MRP2, or MRP5, Luna-Tortós et al. reported that none of the AEDs tested (topiramate, valproate, carbamazepine, phenytoin, levetiracetam, lamotrigine, and phenobarbital) was transported by any of those MRPs [46].

In vivo studies may be needed to confirm the findings from in vitro experiments, but few clinical studies have focused on studying the relationship between AEDs and MRPs.

AED Transport by BCRP. Substrate specificity of BCRP significantly overlaps with that of P-gp. However, the role of BCRP in AED resistance is less well studied in comparison to P-gp or the MRPs [31]. Using BCRP-transfected MDCKII cells, Cerveny et al. [47] reported that none of the tested AEDs (phenobarbital, phenytoin, ethosuximide, primidone, valproate, carbamazepine, clonazepam, and lamotrigine) was transported by BCRP. However, Nakanishi et al. [48] reported that the brain-to-plasma concentration ratio values of phenobarbital, clobazam, zonisamide, gabapentin, tiagabine, and levetiracetam were higher in $m d r 1 a / b /$ Bcrp triple knockout mice than those in $m d r 1 a / b$ double knockout mice, suggesting the involvement of BCRP in the transport of these AEDs.

Current evidence suggests that most AEDs are not transported by BCRP, though discrepancies exist between in vitro and in vivo findings [49].

Efflux Transporter Upregulation Mechanism in Epilepsy. An important question that stems from the transporter hypothesis is whether overexpression of efflux transporters at the blood-brain barrier observed in epilepsy is acquired or constitutive. Current evidence suggests that seizures, genetic factors, or a combination of both are likely to be the major contributors to efflux transporter overexpression at the blood-brain barrier in epilepsy [31]. Experimental data mostly from animal studies support that P-gp upregulation in epileptic regions of the brain occurs mainly as a result of seizure activity. Van Vliet et al. [50] also reported increased MRP1, MRP2, and BCRP protein expression levels in rat astrocytes and cerebral blood vessels after acute status epilepticus and in chronic epilepsy. Similar to the finding with P-gp, overexpression of these transporters was greater in chronic epileptic rats that demonstrated progression of epilepsy.

Recent research in the field has postulated two main mechanisms leading to efflux transporter overexpression in the brain in epilepsy: (1) AED-mediated induction of efflux transporters via nuclear receptors and (2) seizure-induced signaling causing efflux transporter overexpression [31]. Regarding the first mechanism, studies on whether AEDs 
induce efflux transporter overexpression have yielded inconsistent results. However, studies have shown that seizures induce brain capillary P-gp expression levels. If P-gp levels were already maximally induced in the study of Wang-Tilz et al. [51], one would not expect to see additional increases in P-gp expression levels by AEDs. Consistent with this, Wen et al. reported due to AED activation of the ligand-activated transcription factors pregnant $\mathrm{X}$ receptor and/or constitutive androstane receptor [52]. It is important to note that AED-mediated upregulation of drug efflux transporters at the blood-brain barrier and in other tissues does not explain why some patients are resistant to the very first AED they are given. While this speaks against the theory that AEDs are the main cause for drug resistance due to transporter upregulation, it is possible that AEDs are one contributor, among others, to refractory epilepsy [31].

The second mechanism that has been shown to result in increased efflux transporter expression levels is through recurring seizures. In this regard, Lazarowski et al. [53] showed that daily induced seizures result in a progressive increase of P-gp protein expression at the blood-brain barrier, and further will cause resistance. Importantly, resistance to drugs that are not P-gp substrates, such as carbamazepine, diazepam, or levetiracetam was not observed. Therefore, this new model could be useful for screening novel AEDs that are P-gp substrates and have the potential to control seizures in pharmacoresistant epilepsy.

The molecular signaling mechanism underlying increased efflux transporter expression levels in epilepsy has been studied. In this regard, evidence from in vitro and in vivo rodent studies suggests that targeting this pathway could control P-gp expression and activity levels, and thus, help increase AED brain penetration and improve AED efficacy to control seizures in drug-resistant epilepsy. To fully assess if P-gp upregulation has any relevant consequences on pharmacoresistance, studying P-gp expression in brain tissue from both AED-responsive and AED-resistant patients and/ or conducting PET imaging using P-gp substrates or inhibitors in patients would be critical. At present, aspects of the transporter hypothesis are still controversial, and further research is needed to determine the clinical relevance of efflux transporter overexpression at the blood-brain barrier [31].

Pharmacokinetic hypothesis. The pharmacokinetic hypothesis proposes that overexpression of efflux transporters in peripheral organs, such as intestine, liver, and kidney decreases ASD plasma levels in refractory epilepsy patients, thereby reducing the amount of ASD available to cross the blood-brain barrier and reach the epileptic focus in the brain [54].

Alterations in expression and functionality of multidrug transporters in patients with intractable epilepsy need not necessarily be restricted to the brain but could also occur in other tissues, such as the small intestine, where P-glycoprotein is thought to form a barrier against entrance of drugs from the intestinal lumen into the bloodstream, thereby limiting their oral bioavailability. In support of this theory, several studies have reported persistent subtherapeutic plas- ma levels of anticonvulsants (including phenytoin and phenobarbital) despite aggressive and chronic administration of anticonvulsants in patients with drug-resistant epilepsy that has been associated with overexpression of MDR1 [32]. Support for the pharmacokinetic hypothesis comes from studies showing persistently low ASD levels in patients with drug-resistant epilepsy, which, however, relate to drug metabolizing enzymes rather than to efflux transporters such as P-glycoprotein [31]. The metabolism of ASDs is mainly mediated by liver cytochrome P450. Some of the cytochromes of this group have allelic types encoding isoforms which have different activity and, in turn, can affect the concentration of many drugs, including ASDs, in the blood serum. Cytochrome P450 metabolic enzymes not only occur in the periphery, but also in the brain parenchyma and endothelial cells of the blood-brain barrier, thus adding to the barrier function. Changes in the cerebrovascular hemodynamic conditions can affect expression of cytochrome P450 enzymes and multidrug-resistance transporters, leading to a synergistic role in drug resistance [55].

The liver is involved in potential pharmacokinetic changes by overexpression of P-gp, 99mTc-hexakis-2-methoxyisobutylisonitrile, what increased hepatic clearance that could contribute to ASD resistance [56]. The animal studies do not support the pharmacokinetic hypothesis [27]. In addition, data from clinical studies show that ASDresponsive and ASD-resistant patients display adverse events to the same extent, suggesting similar plasma ASD levels in the two groups of patients [32]. One explanation for this observation is that efflux transporter overexpression is restricted to the epileptic focus. This observation also suggests that the same plasma ASD concentrations are due to the same enzyme and transporter expression levels in peripheral organs. While both explanations are plausible, one does not necessarily lead to the other.

Together, the pharmacokinetic hypothesis of refractory epilepsy as a stand-alone theory is difficult to validate. One can argue that because abnormalities in ASD plasma concentrations can be readily captured by therapeutic drug monitoring, pharmacokinetic variability is probably not a major contributor to pharmacoresistance in situations where ASD doses are adjusted accordingly. This argument, however, is further complicated because therapeutic ASD plasma concentrations vary among patients, and no one specific therapeutic ASD concentration range is applicable to all patients.

Neural network hypothesis. Growing body of electrophysiological, neuroimaging, and clinical evidence suggests that epilepsy, including drug-resistant epilepsy, is a network disorder. Recent observations led to the emergence of the neural network hypothesis that might mechanistically explain the development of drug-resistant epilepsy [31]. According to this hypothesis, epilepsy-associated structural and functional alterations lead to an abnormal and maladaptive remodelling of neural networks that become resistant to antiepileptic medication. The underlying substrates of a maladaptive network architecture range from axonal sprouting and synaptic 
reorganization to neurogenesis and gliosis [31]. Structural alterations in both drug-resistant lesional and non-lesional epilepsy are widespread and extend to regions beyond the borders of the epileptogenic focus. Thus, patients with temporal lobe epilepsy show significantly reduced cortical thickness in the supramarginal, middle and upper temporal gyri, temporal pole, insula, cuneus, superior frontal, precentral, posterior cingulate, operculum, lateral orbitofrontal, lingual, upper parietal, postcentral, lower parietal, lateral occipital, paracentral and isthmus cingulate of the right hemisphere [57]. In the left hemisphere, cortical thinning was identified in the supramarginal, precentral, and middle frontal regions. Like structural connectivity, functional connectivity displays distinct and frequency-dependent changes in the interregional neural oscillations. Thus, in theta frequency, connectivity from the temporal and frontal lobes to thalamus initially shows a continuous decrease in the connectivity intensity, later followed by a continuous increase before the spike generation [57]. In contrast, connectivity from the thalamus to the frontal lobe shows an inverted pattern - initial continuous increase in connectivity intensity, followed by a decrease in connectivity [57]. Several factors modulating the topology of neural networks in patients with drugresistant epilepsy have been described. Thus, patients with sleep- and awake-related seizures display different patterns of structural alterations and network organization. Patients with sleep-related seizures compared to those with awakerelated seizures have larger volumes of bilateral insula, superior temporal, and orbitofrontal cortices [58]. Volumes of hippocampus, amygdala, caudate, pallidum, and putamen are larger in patients with sleep-related seizures than in patients with awake-related seizures [58].

Summing up, patterns of brain network reorganization in drug-resistant epilepsy are characterized by an increased segregability, low integrability, and reduced resilience [59]. However, one of the substantial limitations of this hypothesis is that neural network alterations lead to treatment refractoriness not in all epilepsy patients, and therefore, further studies are required to establish the clear-cut network alterations related to drug resistance.

The gene variant hypothesis. In recent years, numerous studies have demonstrated that genetic variation is involved in the drug resistance of epilepsy [60]. Gene variants in transporters, targets as well as metabolizing enzymes, are hypothesized to contribute to drug resistance mechanisms, especially genetic variations found in drug resistance-related genes, including the voltage-dependent sodium and potassium channels genes, and the metabolizer of endogenous and xenobiotic substances genes. Advances in genomic technologies have facilitated the genome-wide discovery of common and rare variants and have increased our understanding of genetics in epilepsy; however, the mechanisms underlying pharmacological resistance have not been fully elucidated. The most frequently studied polymorphisms are those associated with multidrug resistance genes (MDR): ATP-binding cassette subfamily B member 1 (ABCB1 or MDR1) and ATP-binding cassette subfamily $\mathrm{C}$ member 2 (ABCC2 or
MRP2); SCN a subunits 1, 2 and 3 (SCN1, SCN2 and SCN3); and metabolizers of endogenous and xenobiotic substances, cytochromes P450 families 2 and 3 (CYP2 and CYP3) [61]. For example, a meta-analysis on the ABCB1 C3435T polymorphism showed association of CC genotype with drugresistant epilepsy in Caucasians only, while a more recent systematic review showed the TT genotype polymorphism to be correlated [62]. Also, in intractable epilepsy and other mental disabilities, whole-exome sequencing (WES) identified de novo variants in the Bernardinelli-Seip congenital lipodystrophy 2 (BSCL2) gene in two patients [63], or the case of recently reported changes in the KCNQ2 gene which present with both benign seizure disorders and early onset epileptic encephalopathies (EOEE), the latter including patients who present refractory seizures following standard AED treatment and development delay [64].

It is hypothesized that genetic variants may also contribute to the efficacy of drug treatments for epilepsy; for example, adverse or toxic reactions, teratogenic risk in pregnancy, as well as long-term outcomes have been observed among PWE [65]. In recent studies, the association between genetic polymorphisms, treatment responses in epilepsy and antiepileptic drugs (AED) reactions (toxic, adverse or those related with its efficacy) have been investigated.

It was reported that polymorphisms in the human leukocyte antigen (HLA) gene were associated with severe cutaneous adverse AED reactions [66], and polymorphisms in a number of other genes, including $A B C B 1, A B C C 2, G A B R A 6$, GABRG2, CYP2C9, CYP3A4, UDP-glucuronosyltransferase (UGT)1A1, UGT1A4, UGT1A6, UGT2B7, SCN2A and SC$N 1 A$, have been associated with the concentration, response and efficacy of some of the most commonly used AEDs in clinical practice, including carbamazepine, oxcarbazepine, phenytoin, lamotrigine and valproic acid. Esmaeilzadeh et al. reported an association between HLA polymorphisms and severe cutaneous adverse reactions (SCARs) induced by drugs and it was found that the hypersensitivity to different AEDs, including phenytoin, carbamazepine, valproic acid, topiramate and lamotrigine, was associated with $H L A$ $A$ gene polymorphisms [67]. Although this hypothesis proposes an inherent resistance as the cause of intractable seizures, several underlying processes are also involved in epileptic seizures including microglial activation, glutamateinduced excitotoxicity, mitochondrial dysfunction, oxidative stress, and the formation of reactive oxygen species [60]. U1timately, this requires simultaneous malfunction of several enzymes, proteins, channels, and receptors [68].

Consequently, it is difficult to believe that an individual is born with such a vast number of gene polymorphisms to the extent that both pharmacokinetic and pharmacodynamic mechanisms are hindered. Overall drug resistant patients are a highly heterogeneous group and no theory in isolation can explain multi-drug inefficacy in every patient [69].

The epigenetic hypothesis. The genome is one source of endogenous variation, contributing to different disease risks between different people. There are, however, other sources of variation, such as "omes" beyond the genome: the 
epigenome, transcriptome, proteome, microbiome, and so on. Some of these "omes" have been interrogated for their role in drug resistance in epilepsy, but it must be acknowledged at this point that the data available are even more sparse than for most genome-based studies and that, currently, none of these "omes" have been proven to influence drug resistance [27]. Studying epigenomic contribution to drug resistance in epilepsy [70], which is likely to be due to processes in the brain, is very challenging.

Epigenetic mechanisms have also been proposed to explain the development of pharmacoresistance in epilepsy patients, influencing the sustained patterns of gene expression that regulate AED uptake and mechanism of action. In the future, drugs inhibiting DNA methyltransferases (DNMTs) and histone lysine deacetylases (KDACs) could provide new treatments for patients unaffected by currently available anti-epilepsy medications. Not only are drugs acting on epigenetic processes currently available and in development for a variety of human diseases, but there is some evidence that one or more commonly used AEDs may act, in part, through epigenetic mechanisms. Most of the work identifying epigenetic changes in epilepsy has focused on DNA methylation. Recently, there has been exceptional interest in the role of microRNAs, especially where attention has also focused on their potential for use as biomarkers to support early diagnosis and prognosis in the clinic. In contrast, other aspects, such as histone modifications and long noncoding RNAs, have been less studied [71].

Among classes of molecules constituting the epigenome are histones and noncoding RNAs, both long and shorter, including microRNAs. The latter contribute to RNA silencing and post-transcriptional regulation of gene expression, altering expression levels of multiple proteins. A central problem in studying the epigenome in humans is to disentangle cause from effect and relevance either way from epiphenomena [27]. The mechanisms of action of ketogenic diet in epilepsy have been revealed recently, such as epigenetic mechanism for increase the adenosine level in the brain and inhibition of DNA methylation. Thus, although a series of microRNAs have been shown to associate with human TLE [72], the studied tissue had been surgically resected from people with drug resistance, and cause and effect (for either disease susceptibility or drug resistance) could not be distinguished. In animal models, manipulation of specific microRNAs can influence seizures and disease [73], though some data are less supportive, however, whether this would be the case in human epilepsy, and specifically whether this approach would counter drug resistance, remains unknown.

Neuroinflammatory hypothesis. Accumulating evidence overthepastdecadeindicatesanimportantpathophysiological role of brain inflammation in pharmacoresistant epilepsy. Different inflammatory molecules and pathways have been shown to significantly contribute to the mechanisms of seizure generation and progression in different experimental models [74]. Inflammation refers to the complex biological response of tissues against infections or sterile (noninfectious) injuries, it is closely associated with the activation of both innate and adaptive immune cells. It represents a key homeostatic mechanism of the body's defense, which is crucial for activating mechanisms for tissue repair, via the production of a large array of inflammatory cytokines and related effector molecules. A novel emerging concept in inflammation is the specific interaction between the innate immune system and injured brain tissue, known as neuroinflammation. Although neuroinflammation currently lacks a consensus definition, in general it can be seen as the biosynthesis and release of molecules with inflammatory properties by resident cells of the brain, including activated microglia and astrocytes, neurons, endothelial cells of the blood-brain barrier, and blood-born macrophages.

A notable finding is that the inflammatory mediators released by the resident brain cells during epileptic activity (i.e. cytokines, chemokines, alarmins/danger signals, prostaglandins, complement factors) are not only effectors molecules of the immune system promoting local inflammation, but also function as neuromodulators directly affecting neuronal function and excitability [75]. Prominent changes in several immune/inflammatory pathways, such as IL-1R1/ TLR4, COX-2, TNF-a, complement and chemokines have been reported within epileptogenic lesions in preclinical and clinical studies. These pathways seem critically involved in ictogenesis and epileptogenesis. A more detailed assessment of the use of these inflammatory pathways is needed as potential biomarkers which contribute to the development of epilepsy or to measure the effectiveness of therapeutic interventions.

Gut-brain axis. The "gut microbiota-brain axis" is the interaction between gut microbiome and brain. This bidirectional communication is provided by the nervous, endocrine, immune, circulatory, and metabolic pathways [76]. Ketogenic Diet (KD) may be considered another alternative treatment in drug-resistant epilepsy (DRE). This type of diet was used since 1921, in the treatment of intractable epilepsy in children.

Animal models. Olson et al., demonstrated in two-seizure mouse models, KD changes microbiota and leads to anti-seizure protection. KD fed-mice have demonstrated a decreasing in seizure duration and frequency. Also, it was measured the level of GABA and glutamate. GABA levels were higher in $\mathrm{KD}$ fed-mice. The direct association between chronic stress-induced epilepsy and intestinal dysbiosis in SpragueDawley rats was reported in a study [77].

$K D$, microbiome and epilepsy. KD is a high-fat, and very low-carbohydrate diet. KD in children with retractable epilepsy was evaluated in randomized controlled prospective study. Patients had a relevant decreasing in seizure severity. Seizures were reduced to at least $50 \%$ in children who received KD. The effect of the KD on gut microbiome in children with DRE, due to GLUT-1 deficiency was investigated for 90 days. Compared with baseline increasing in Desulfovibrio species was established after KD. There were no significant differences in Bacteroidetes and Firmicutes levels. Xie et al., used KD (4:1 ratio) for a week. They noticed that $64 \%$ of children with DRE had $\geq 50 \%$ decreasing in seizure frequen- 
cy. Compared with baseline, while Bacteroides increased markedly, Bifidobacterium increased less significantly after KD therapy. Cronobacter, which was at high levels before $\mathrm{KD}$, after therapy presented similar levels with the control group [78]. KD (4:1 ratio) was used in children (five patients had Dravet syndrome, three each had West syndrome and Lennox-Gastaut syndrome, and the remainder couldn't be classified) for six months. Reduction of Firmicutes and increasing Bacteroidetes levels were reported after therapy. KD-non-responder group demonstrated increased levels of Clostridiales, Ruminococcaceae, Rikenellaceae, Lachnospiraceae, and Alistipes, comparative with responder group [79]. Lindefeldt et al., evaluated the effect of KD on eleven children with DRE (due to perinatal asphyxia, encephalitis, cortical dysplasia, tuberous sclerosis, and unknown cause) and one with pyruvate dehydrogenase deficiency. Relative abundance of Escherichia coli increasing was observed on KD. Levels of Bifidobacteria as well as Eubacterium rectale and Dialister were decreased during KD therapy [80].

Gut dysbiosis in epilepsy patients. Probiotics show beneficial effect on seizures in patients with DRE. In a pilot study was shown seizures reduction and higher quality of life in $28.9 \%$ of adult patients with DRE, who were treated with probiotic cocktail [81]. Another study demonstrated that rotavirus infection manifests a risk factor for neonatal seizures. Probiotic administration after birth was linked with reduced risk of seizures [82]. Peng et al, established that Bacteroidetes phylum is the largest one (56.7\% in Drug Sensitive (DS) and $57.2 \%$ in Healthy Control (HC)); Firmicutes phylum is the second dominant ( $38.2 \%$ in DS and $37.5 \%$ in $\mathrm{HC}$ ). The while, Bacteroidetes was relatively lower (45.7\%), and the Firmicutes was the largest phylum $(46.9 \%)$ in patients with DRE. Also, it was shown an increasing of other rare phyla in the DRE group; Verrucomicrobia was more abundant in the DRE group $(0.32 \%)$ than $(0.03 \%$ in DS) and $(0.09 \%$ in $\mathrm{HC}$ ) groups. Patients with DRE have altered composition of gut microbiota; so, dysbiosis may be implicated in the pathophysiology of DRE.

\section{Biomarkers of drug-resistant epilepsy}

Biomarkers may play a role in individualized epileptic treatment, based on the patients' biomarker profile. Molecular biomarkers, inflammatory markers can indicate not only the presence, type, and severity of neuropathologically damaged tissue with epileptogenic potential, but may also have the potential for localizing epileptogenic zone (EZ) [83].

Metabolites, proteins, messenger RNAs (mRNAs) and microRNAs (miRNAs) play a critical role in the regulation of neuronal biological processes, as well they might have importance in early disease diagnosis, effective prognostic monitoring and can be clubbed with existing techniques to preoperatively localize the EZ. Thus, the challenge is to find a biomarker which one can help accurately localize the EZ and offering new therapeutic approach strategies. Several microarray and target studies have reported a differential expression of more than 100 miRNAs in epilepsy. From those, miR-146a, miR-155 and miR-132 have a key role in epilepsyrelated biological processes, such as neuroinflammation, neuronal growth, neuroprotection and neurodegeneration [84]. MiRNA-155 has been shown, in experimental studies, to be much higher in patients with generalized genetic epilepsy compared to control, and miRNA-4521 could serve as a potential biomarker in refractory epilepsy. Changes in the mRNA levels of various glutamate and gammaaminobutyric acid (GABA) receptor subunits modulating of glutamatergic and GABAergic synaptic transmission, also mRNA polyadenylation profile can be utilized as a biomarker in generalized, and focal epilepsies [85]. Inflammatory molecules have been identified in experimental models of epilepsy and surgically resected brain tissue from treatmentresistant epilepsy. Different components especially, cyclooxygenase-2 (COX-2) play an important role in the post seizure inflammation, possibly contributing to secondary damage in the brain and the increased likelihood of repetitive seizures. High mobility group box-1 (HMGB1), that takes part in the immune response via activating macrophages and endothelial cells, leading to the release of tumor necrosis factor-a (TNF-a), interleukin-1 (IL-1), interleukin-6 (IL-6) has been indicated as a potential therapeutic agent in epilepsy and as a non-invasive biomarker, which could identify patients with high risk of epilepsy. The level of HMGB1 has been shown to increase within 3-4 h after seizure, proving HMGB1 to be a promising marker. Moreover, it is known that in drug-resistant epilepsy (DRE) resulting from Rasmussen's encephalitis, some patients showed seizure improvement following adalimumab administration, an anti-TNF-a therapy [86].

In sum, biomarker discovery and validation efforts need to balance between curation and control on the one hand, while allowing for broader coverage and generalizability on the other [87]. In time, this will lead to the development of effective strategies for the early screening, clinical diagnosis, treatment, and prevention of refractory epilepsy.

\section{Advances in pharmacological treatment}

Several antiepileptic drugs are currently in clinical trials whose mode of action is either historical, novel or adapted from previous AEDs. Everolimus, a compound which had been in use primarily for other clinical indications (renal cancer, organ transplant immunosuppression, metastatic neuroendocrine tumors) was approved in 2018 and has shown promise as an antiepileptogenic agent in patients with tuberous sclerosis. It works as an inhibitor of an overactive and dysregulated mTOR pathway. EXIST-3 trial [88] showed response rate of $28.2 \%$ and $40.0 \%$ for low-exposure and highexposure everolimus, respectively, compared to placebo of $15.1 \%$. Other compounds already in use for other medical indications and being tested in epilepsy include melatonin, biperiden, fenfluramine, bumetanide and verapamil [89]. Apart from these clinical in-use medications, drugs specifically designed for epilepsy are under investigation.

Clinical studies of cenobamate, which was recently approved for the treatment of partial-onset seizures in adults, showed approximately $20 \%$ of patients experienced seizure freedom, which is very impressive compared with previous add-on clinical trials with various other novel AEDs in pa- 
tients with DRE [90]. A similar impressive antiseizure effect has been observed with the novel ASD fenfluramine in Dravet syndrome, in which approximately $25 \%$ of patients had long-term seizure freedom, suggesting that the long hopedfor breakthrough is a feasible goal [91].

Other compounds undergoing recruitment in phase $2 / 3$ studies (registered on Clinicaltrials.gov) include padsevonil, neurosteroids (ganoxolone), XEN1101, E2082 and vorinostat. Several other drugs appear to be suspended due to failed early stage efficacy studies and others will need further preliminary data to assess their potential [89]. Cannabidiol studies have had a surge of interest after successes in recent trials of patients with Dravet and Lennox-Gastaut (LGS) syndromes and further open-label study has shown good retention rate, efficacy and safety data [92].

Epidiolex is the first canabidiol oral solution to be FDA (Food and Drug Administration) approved. Double-blind controlled studies showed significant seizure reduction in the range of $17 \%-23 \%$ compared to placebo for monthly convulsive seizures in Dravet syndrome and monthly total seizure reduction in the two pivotal LGS trials [93]. Overall, most of the drugs in the pipeline have similar mechanism of actions to traditional drugs. Clinical benefits over existing drugs are, therefore, unlikely in the near future. Alongside the novelty in drug therapy explored by pharmaceutical companies, viral-vector mediated gene therapy has demonstrated success in animal models of focal neocortical epilepsy using chemical-genetics and optogenetics, but no human trials are yet in progress.

Gene therapy. The use of novel therapeutic approaches in the management of epilepsy is steadily progressing. Neurological disorders are sometimes caused by inherited or acquired genetic changes that lead to abnormal nervous system development, neurodegeneration, or impaired neuronal function. About $30 \%$ of the epilepsies have been thought to have a genetic origin [94]. Gene therapy, as an emerging and novel therapeutic approach has curative potentials of the most common neurological disorders including DRE. It had been formerly defined as a method to replace the defective copy of a gene with a normal copy which acts correctly in the cells [95]. There are various methods to transfer and express a gene in a particular region of the brain that include cell transplantation, liposomes, non-viral and viral vector delivery, (adenovirus, adeno-associated virus, herpes simplex virus, lentivirus, and retrovirus). Gene therapy for DRE treatment aims to induce the local release of anticonvulsant or antiepileptogenic properties to counterbalance between excitation and inhibition in the brain. It offers the possibility of targeting therapeutic genes expressing in the seizure generating area without needing tissue ablation. These results are very promising; however, it is important to note that only infrequent types of epilepsy are caused by a single mutant gene; while they are commonly caused by inheritance of two or more susceptibility genes that usually influence a large part of the brain. Therefore, an extensive gene transfer is needed; however, the presently available gene therapies provide only local effects [94]. Improvement in the gene therapy-treating epilepsy can be possible through progress in understanding the disease mechanisms, designing suitable gene vectors, selecting suitable genes, and choosing the right delivery methods. Taken together, gene therapy can be considered as a therapeutic approach in the management of epilepsy; but further studies are needed to verify the safety and efficacy of this method in human. Gene therapy is a more challenging and complicated process than the simple concept of gene replacement. Techniques for transferring of exogenous genes into the desired sequence of target cells have been remarkably improved. Clustered regularly interspaced short palindromic repeats (CRISPR) are types of nucleic acid sequences that function in harmony with CRISPR-associated (Cas) proteins to provide immunity in bacteria and archaea against foreign invasion of nucleotides, such as viruses, plasmid, and phages. CRISPR-Cas9 system has recently attracted increasing attention for therapeutic applications [96]. This system has presented a novel approach in repairing gene defects for treating various types of disorders including neurological diseases. Due to its potential in targeted gene editing and repairing of genetic mutations, CRISPR-Cas9 can also be considered for applying as a possible therapeutic approach in treating DRE with genetic origins. Although it is the most powerful and useful technique for the multiplexed genome manipulating, there are still some challenges regarding efficiency and accuracy concerns, and further studies are needed to verify its safety before clinical applications. Several studies with the aim of epilepsy treating have applied various cell types, such as neural stem cells, mesenchymal stem cells, hippocampal precursor cells, GABAergic precursor cells, and bone marrow-derived mononuclear cells. They all aim to diminish seizure severity and frequency in the brain through different mechanisms of action. Although the results are encouraging, to use cell therapy in clinical application, additional and vigorous studies are necessary to test safety and efficacy of this approach [97].

Exosome therapy. The exosome is a kind of extracellular vesicles which have initially been recognized in the 1980s. Exosomes are characterized by homogeneous shaped nano $(40-100 \mathrm{~nm})$ membranous vesicles with a density of $1.13-1.19 \mathrm{~g} / \mathrm{cm}^{2}$. They can be secreted by several body cell types and have been detected in the various biological fluids including blood, urine, saliva, CSF, breast milk, amniotic fluid, malignant ascites, bronchoalveolar fluid, and synovial fluid. Depending on their source cells, exosomes can contain a variety of lipids, proteins, and genetic elements, such as DNA, non-coding RNAs, mRNAs, and microRNAs. These agents which are also known as "cargo" can be delivered to the surrounding cells or transferred to other distal cells and alter the recipient cell function. Therefore, exosomes can be considered as a novel form of intercellular communication. Interestingly, it has been shown that many cells of the nervous system, such as neurons, microglia, astrocytes, oligodendrocytes, and neural stem cells can release exosomes. Thus, exosomes may have a role in the function, development, and pathologies of the nervous system [98]. Emerging pieces of evidence have suggested that exosomes can be 
used to rescue neuronal pathologies and alterations. It has been revealed that these vesicles have crucial roles in the regeneration process and repair of the nervous system. Their simple structure, low immunogenicity, and ability to cross the blood-brain barrier (BBB) have made a great opportunity to engineer and apply them as vehicles for delivering microRNAs, drugs, proteins, and other active agents to the brain. MicroRNAs have been known as master regulators of gene expression. In the nervous system, they can alter the regulation of various proteins which associate with several neuronal processes and actions. They have been shown to regulate essential genes involved in seizure susceptibility. Therefore, exosomes contained the agents which have antiseizure activity can be potentially applied to manage and treat epilepsy. However, more studies are needed to address their use in clinical trials $[84,98]$.

\section{Neuromodulation in pharmacoresistant epilepsy}

Correctly selected antiepileptic drugs (AEDs) show good clinical improvement in most individuals with epilepsy, although almost a third of these patients at some point will present an inadequate or insufficient response to current AEDs [99]. In individuals with drug-resistant epilepsy the percentage of general morbidity and mortality is significantly higher when compared to drug responsive cases. Therefore, there is a consistent need for improving therapeutically management in this population. From a pathophysiological point of view, epilepsy is a result of abnormal neuronal network activity in the brain due to a pathological increase in excitatory synapses (Glutaminergic) with a decrease in inhibitory activity (GABA-ergic). Thus, approaching this paradigm it is assumed that inhibitory neuromodulation could induce the phenomenon of LTD (long-term excitatory depression) with normalization of the excitation threshold in hyperactive areas and as a result obtain the expected therapeutic effect. Modulation of brain activity can be achieved by impacting specific intra/ extracranial targets, either the peripheral elements of sensitization or direct cortical-subcortical activity as shown in Figure 1. By influencing the activity of these components, a modulation of either widespread brain networks or direct modulation of network nodes can be achieved.

Vagal nerve stimulation (VNS) involves intermittent electrical stimulation of the afferent fibers of the left cervical vagus nerve in a transcutaneous (tVNS) or non-invasive (nVNS) way or by means of an implanted helical electrode connected to a pulse generator. The therapeutic efficacy of VNS appears to be mediated by the activation of fast myelinated fibers in the vagus nerve. Its anticonvulsant effect can be explained by several mechanisms, such as the modulation of neurotransmitter expression with increased inhibition and reduced excitability, changes in cerebral blood flow, desynchronization of electroencephalographic (EEG) rhythms, and anti-inflammatory effects mediated by norepinephrine [100].

Transcranial direct current stimulation (tDCS) is a noninvasive method that modulates cortical excitability using a weak constant electric current passing through two elec- trodes (anode and cathode) applied over the skull. Cortical excitability may increase following anodal stimulation, while it generally decreases after cathodal stimulation. Considering this principle, hyperpolarization using cathodal tDCS has been proposed as therapy to suppress epileptiform discharges [101].

Trigeminal nerve stimulation (TNS) similar to VNS and tDCS uses electrical stimuli but targets trigeminal sensory roots within the facial tissue (ophthalmic nerve, supratrochlear nerve, infraorbital nerve). Considering the projections of the trigeminal pathway, it is thought to generate an arousal-like effect from stimulation of the reticular activating system which leads to a shift of cortical activity from a "synchronized" state to a "desynchronized" state. As seizures present highly synchronous activity, it was hypothesized that desynchronization of the neocortex would have an anticonvulsant effect [102].

Transcranial magnetic stimulation (TMS) is a non-invasive brain stimulation technique which using electromagnetic induction phenomenon is able to module electrical activity of targeted cortical areas. The application of low-frequency $(<1 \mathrm{~Hz})$ repetitive TMS or continuous theta burst stimulation (cTBS) modulates the cortical excitability and produces its relatively long-term depression-like mechanisms in the cortex [103].

Deep brain stimulation (DBS) is an invasive neuromodulation technique that uses electrical stimulation through electrode implantation within the anterior nucleus of thalamus, centromedian nucleus of thalamus, cerebellum, hippocampus, or subthalamic nucleus to interfere with neuronal synchronized oscillations, thus inducing modulation of pathological neural networks [104].

Responsive neurostimulation (RNS) is a closed loop, invasive brain stimulation method that aims to suppress ictal activity by delivering stimulation directly in response to electrographic activity; it is highly time- and area-specific, therefore providing stimulation only when needed [100] (fig. 1).

By reducing cortical excitability, targeted neuromodulation has enormous therapeutic potential in the treatment of epilepsy, especially in drug-resistant epilepsy.

\section{Perspectives}

The issue of drug resistance to epilepsy remains relevant to date. Thus, the use of an integrated approach, personalizing the therapy of patients with refractory epilepsy, can achieve significant positive results.

Therefore, a therapeutic strategy for drug-resistant epilepsy should be based on the one hand, on the suppression of epileptogenesis and, on the other hand, on overcoming drug resistance. An integrated approach to the problem of drug resistance will allow not only obtaining new data on the mechanisms of the pathogenesis of epilepsy, but also improving the algorithm for treating patients and increasing its effectiveness.

Although the previous decades have noted remarkable developments in the neurosciences, technological improvements in diagnostics (genetics, imaging, electrophysiology) 


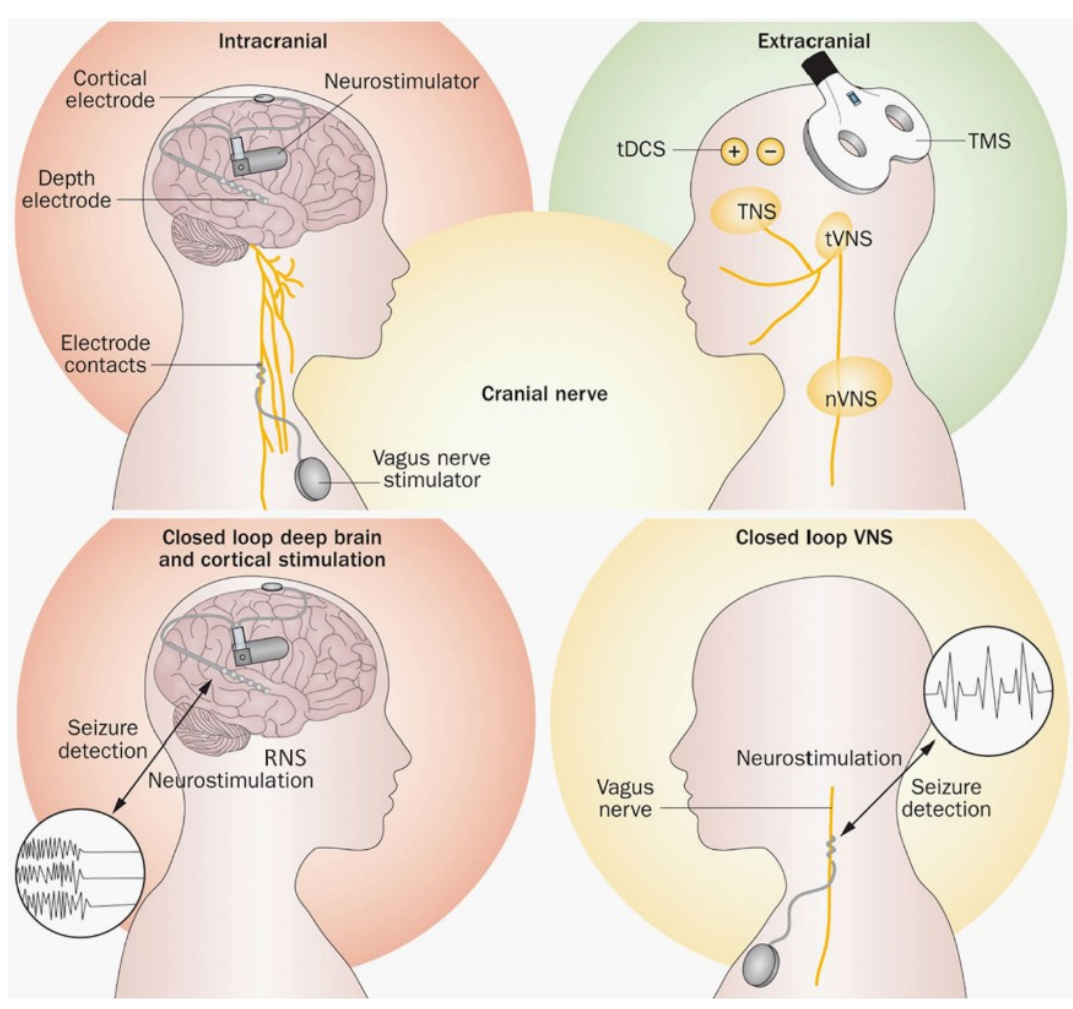

Fig. 1. Types of neurostimulation and targets used for achieving neuromodulatory effect in patients with epilepsy. Adapted from [100]. Transcranial direct current stimulation (tDCS); Trigeminal nerve stimulation (TNS); Transcutaneous vagus nerve stimulation (tVNS); Non-invasive vagus nerve stimulation (nVNS); Transcranial magnetic stimulation (TMS); Deep brain stimulation (DBS); Responsive neurostimulation (RNS)

and increased options of drug therapies, epidemiological studies suggest that this has not translated in wide benefits for drug-resistant patients [105].

\section{Conclusions}

Neuroscience of drug-resistant epilepsy faces many challenges. Inflammatory mediators, biomarkers, and genes might allow the identification of new treatment targets, contribute to an earlier diagnosis, and assess the clinical outcomes. New therapeutic approaches, with personalized therapy for each patient, offer better perspectives for patients and their families. Thus, drug resistance in epilepsy is an urgent scientific and practical issue that requires fundamental and interdependent clinical research. The further development of new surgical methods and non-surgical treatment of drug-resistant epilepsy remains relevant, including the modernization of neurorehabilitation methods.

\section{References}

1. Fisher RS, Acevedo C, Arzimanoglou A, et al. ILAE official report: a practical clinical definition of epilepsy. Epilepsia. 2014;55(4):475-82. doi: 10.1111/epi.12550.

2. Beghi E, Giussani G, Nichols E, et al. Global, regional, and national burden of epilepsy, 1990-2016: a systematic analysis for the Global Burden of Disease Study 2016. Lancet Neurol. 2019;18(4):357-375. doi: 10.1016/S1474-4422(18)30454-X

3. Beghi E. The epidemiology of epilepsy. Neuroepidemiology. 2020;54(Suppl 2):185-191. doi: 10.1159/000503831.
4. Shankar R, Rowe C, Van Hoorn A, et al. Under representation of people with epilepsy and intellectual disability in research. PLoS One. 2018;13(6):e0198261. doi: 10.1371/journal.pone.0198261.

5. Stangl AL, Earnshaw VA, Logie CH, et al. The Health Stigma and Discrimination Framework: a global, crosscutting framework to inform research, intervention development, and policy on health-related stigmas. BMC Med. 2019;17(1):31. doi: 10.1186/s12916-019-1271-3.

6. Tedrus GMAS, Pereira RB, Zoppi M. Epilepsy, stigma, and family. Epilepsy Behav. 2018;78:265-268. doi: 10.1016/j.yebeh.2017.08.007.

7. Chahal CAA, Salloum MN, Alahdab F, et al. Systematic review of the genetics of Sudden Unexpected Death in Epilepsy: potential overlap with sudden cardiac death and arrhythmia-related genes. J Am Heart Assoc. 2020;9(1):e012264. doi: 10.1161/JAHA.119.012264.

8. Lhatoo S, Langan Y, Sander J. Sudden unexpected death in epilepsy. Postgrad Med J. 1999;75(890):706-709. doi: 10.1136/pgmj.75.890.706.

9. Téllez-Zenteno JF, Ronquillo LH, Wiebe S. Sudden unexpected death in epilepsy: evidence-based analysis of incidence and risk factors. Epilepsy Res. 2005;65(1):101-115. doi: 10.1016/j.eplepsyres.2005.05.004.

10. Walczak T, Leppik IE, D’Amelio M, et al. Incidence and risk factors in sudden unexpected death in epilepsy: a prospective cohort study. Neurology. 2001;56(4):519-525. doi: 10.1212/wnl.56.4.519.

11. Kwan P, Brodie MJ. Effectiveness of first antiepileptic drug. Epilepsia. 2001;42(10):1255-1260. doi: 10.1046/j.1528-1157.2001.04501.x.

12. Brodie M, Barry SJ, Bamagous GA, et al. Patterns of treatment response in newly diagnosed epilepsy. Neurology. 2012;78(20):1548-1554. doi: 10.1212/WNL.0b013e3182563b19.

13. Kwan P, Arzimanoglou A, Berg AT, et al. Definition of drug-resistant epilepsy: consensus proposal by the ad hoc Task Force of the ILAE Commission on Therapeutic Strategies. Epilepsia. 2010;51(6):10691077. doi: 10.1111/j.1528-1167.2009.02397.x.

14. Leppik I. Intractable epilepsy in adults. Epilepsy Res Suppl. 1992;5:7-11. 
15. Jallon P. The problem of intractability: the continuing need for new medical therapies in epilepsy. Epilepsia. 1997;38 Suppl 9:S37-S42. doi: 10.1111/j.1528-1157.1997.tb05203.x.

16. Arzimanoglou A, Ryvlin P. Towards a clinically meaningful definition of drug-resistance. In: Kahane P, Berg A, Loscher W, et al., editors. Drug-Resistant Epilepsy. Montrouge: John Libbey Eurotext; 2008. p. 1-6.

17. Malek N, Heath CA, Greene J. A review of medication adherence in people with epilepsy. Acta Neurol Scand. 2017;135(5):507-515. doi: 10.1111/ane.12703.

18. Nakken KO, Kostov H, Ramm-Pettersen A, Heminghyt E, Bakke SJ, Nedregaard B, Egge A. [Epilepsy surgery: assessment and patient selection]. Tidsskr Nor Legeforen. 2012;132(14):1614-8. Norwegian. doi: 10.4045/tidsskr.11.1149.

19. Lerche H. Drug-resistant epilepsy - time to target mechanisms. Nat Rev Neurol. 2020;16(11):595-596. doi: 10.1038/s41582-020-00419-y.

20. Rogawski MA. The intrinsic severity hypothesis of pharmacoresistance to antiepileptic drugs. Epilepsia. 2013;54:33-40. doi: 10.1111/epi.12182.

21. Schmidt D, Löscher W. New developments in antiepileptic drug resistance: an integrative view. Epilepsy Curr. 2009;9(2):47-52. doi: 10.1111/j.1535-7511.2008.01289.x.

22. Brodie MJ. Road to refractory epilepsy: the Glasgow story. Epilepsia. 2013;54 Suppl 2:5-8. doi: 10.1111/epi.12175.

23. Marson A, Jacoby A, Johnson A, et al. Immediate versus deferred antiepileptic drug treatment for early epilepsy and single seizures: a randomised controlled trial. Lancet. 2005;365(9476):2007-2013. doi: 10.1016/S0140-6736(05)66694-9.

24. Remy S, Beck H. Molecular and cellular mechanisms of pharmacoresistance in epilepsy. Brain. 2006;129(1):18-35. doi: 10.1093/brain/awh682.

25. Rogawski MA, Löscher W, Rho JM. Mechanisms of action of antiseizure drugs and the ketogenic diet. Cold Spring Harb Perspect Med. 2016;6(5):a022780. doi: 10.1101/cshperspect.a022780.

26. Remy S, Gabriel S, Urban BW, et al. A novel mechanism underlying drug resistance in chronic epilepsy. Ann Neurol. 2003;53(4):469-479. doi: 10.1002/ana.10473.

27. Löscher W, Potschka H, Sisodiya SM, et al. Drug resistance in epilepsy: clinical impact, potential mechanisms, and new innovative treatment options. Pharmacol Rev. 2020;72(3):606-638. doi: 10.1124/ pr.120.019539.

28. Remy S, Urban BW, Elger CE, et al. Anticonvulsant pharmacology of voltage-gated $\mathrm{Na}+$ channels in hippocampal neurons of control and chronically epileptic rats. Eur J Neurosci. 2003;17(12):2648-2658. doi: 10.1046/j.1460-9568.2003.02710.x.

29. Doeser A, Dickhof G, Reitze M, et al. Targeting pharmacoresistant epilepsy and epileptogenesis with a dual-purpose antiepileptic drug. Brain. 2015;138(2):371-387. doi: 10.1093/brain/awu339.

30. Brooks-Kayal AR, Shumate MD, Jin H, et al. Selective changes in single cell GABA A receptor subunit expression and function in temporal lobe epilepsy. Nature Med. 1998;4(10):1166-1172. doi: 10.1038/2661.

31. Tang F, Hartz A, Bauer B. Drug-resistant epilepsy: multiple hypotheses, few answers. Front Neurol. 2017;8:301. doi: 10.3389/fneur.2017.00301.

32. Loscher W, Luna-Tortós C, Römermann K, et al. Do ATP-binding cassette transporters cause pharmacoresistance in epilepsy? Problems and approaches in determining which antiepileptic drugs are affected. Curr Pharm Des. 2011;17(26):2808-2828. doi: 10.2174/138161211797440212.

33. Sun Y, Luo X, Yang K, et al. Neural overexpression of multidrug resistance-associated protein 1 and refractory epilepsy: a meta-analysis of nine studies. Int J Neurosci. 2016;126(4):308-317. doi: 10.3109/ 00207454.2015.1015724.

34. Aronica E, Gorter JA, Ramkema M, et al. Expression and cellular distribution of multidrug resistance-related proteins in the hippocampus of patients with mesial temporal lobe epilepsy. Epilepsia. 2004;45(5):441451. doi: 10.1111/j.0013-9580.2004.57703.x.

35. Zhang C, Kwan P, Zuo Z, et al. The transport of antiepileptic drugs by Pglycoprotein. Adv Drug Deliv Rev. 2012;64(10):930-942. doi: 10.1016/j. addr.2011.12.003.
36. Zhang C, Kwan P, Zuo Z, et al. In vitro concentration dependent transport of phenytoin and phenobarbital, but not ethosuximide, by human P-glycoprotein. Life Sci. 2010;86(23-24):899-905. doi: 10.1016/j. lfs.2010.04.008.

37. Löscher W, Potschka H. Role of drug efflux transporters in the brain for drug disposition and treatment of brain diseases. Prog Neurobiol. 2005;76(1):22-76. doi: 10.1016/j.pneurobio.2005.04.006.

38. Tishler DM, Weinberg KI, Hinton DR, et al. MDR1 gene expression in brain of patients with medically intractable epilepsy. Epilepsia. 1995;36(1):1-6. doi: 10.1111/j.1528-1157.1995.tb01657.x.

39. Clinckers R, Smolders I, Meurs A, et al. Quantitative in vivo microdialysis study on the influence of multidrug transporters on the blood-brain barrier passage of oxcarbazepine: concomitant use of hippocampal monoamines as pharmacodynamic markers for the anticonvulsant activity. J Pharmacol Exp Ther. 2005;314(2):725-731. doi: 10.1124/ jpet.105.085514.

40. Owen A, Pirmohamed M, Tettey JN, et al. Carbamazepine is not a substrate for P-glycoprotein. Br J Clin Pharmacol. 2001;51(4):345-349. doi: 10.1046/j.1365-2125.2001.01359.x.

41. Baltes S, Fedrowitz M, Tortós CL, et al. Valproic acid is not a substrate for P-glycoprotein or multidrug resistance proteins 1 and 2 in a number of in vitro and in vivo transport assays. J Pharmacol Exp Ther. 2007;320(1):331-343. doi: 10.1124/jpet.106.102491.

42. Luna-Tortós C, Fedrowitz M, Löscher W. Several major antiepileptic drugs are substrates for human P-glycoprotein. Neuropharmacology. 2008;55(8):1364-1375. doi: 10.1016/j.neuropharm.2008.08.032.

43. Marchi N, Guiso G, Rizzi M, et al. A pilot study on brain-to-plasma partition of 10, 11-dyhydro-10-hydroxy-5H-dibenzo (b, f) azepine5-carboxamide and MDR1 brain expression in epilepsy patients not responding to oxcarbazepine. Epilepsia. 2005;46(10):1613-1619. doi: 10.1111/j.1528-1167.2005.00265.x.

44. Potschka H, Baltes S, Löscher W. Inhibition of multidrug transporters by verapamil or probenecid does not alter blood-brain barrier penetration of levetiracetam in rats. Epilepsy Res. 2004;58(2-3):85-91. doi: 10.1016/j. eplepsyres.2003.12.007.

45. Baltes S, Gastens AM, Fedrowitz M, et al. Differences in the transport of the antiepileptic drugs phenytoin, levetiracetam and carbamazepine by human and mouse P-glycoprotein. Neuropharmacology. 2007;52(2):333-346. doi: 10.1016/j.neuropharm.2006.07.038.

46. Luna-Tortós C, Rambeck B, Jürgens UH, et al. The antiepileptic drug topiramate is a substrate for human P-glycoprotein but not multidrug resistance proteins. Pharm Res. 2009;26(11):2464-2470. doi: 10.1007/ s11095-009-9961-8.

47. Cerveny L, Pavek P, Malakova J, et al. Lack of interactions between breast cancer resistance protein (bcrp/abcg2) and selected antiepileptic agents. Epilepsia. 2006;47(3):461-8. doi: 10.1111/j.1528-1167.2006.00453.x.

48. Nakanishi H, Yonezawa A, Matsubara K, et al. Impact of P-glycoprotein and breast cancer resistance protein on the brain distribution of antiepileptic drugs in knockout mouse models. Eur J Pharmacol. 2013;710(13):20-28. doi: 10.1016/j.ejphar.2013.03.049.

49. Römermann K, Helmer R, Löscher W. The antiepileptic drug lamotrigine is a substrate of mouse and human breast cancer resistance protein (ABCG2). Neuropharmacology. 2015;93:7-14. doi: 10.1016/j. neuropharm.2015.01.015.

50. Van Vliet EA, Redeker S, Aronica E, et al. Expression of multidrug transporters MRP1, MRP2, and BCRP shortly after status epilepticus, during the latent period, and in chronic epileptic rats. Epilepsia. 2005;46(10):1569-1580. doi: 10.1111/j.1528-1167.2005.00250.x.

51. Wang-Tilz Y, Tilz C, Wang B, et al. Influence of lamotrigine and topiramate on MDR1 expression in difficult-to-treat temporal lobe epilepsy. Epilepsia. 2006;47(2):233-239. doi: 10.1111/j.1528-1167.2006.00414.x.

52. Wen T, Liu YC, Yang HW, et al. Effect of 21-day exposure of phenobarbital, carbamazepine and phenytoin on P-glycoprotein expression and activity in the rat brain. J Neurol Sci. 2008;270(1-2):99-106. doi: 10.1016/j.jns.2008.02.016. 
53. Lazarowski A, Ramos AJ, García-Rivello H, et al. Neuronal and glial expression of the multidrug resistance gene product in an experimental epilepsy model. Cellular and molecular neurobiology. 2004;24(1):77-85. doi: 10.1023/b:cemn.0000012726.43842.d2.

54. Lazarowski A, Czornyj L, Lubienieki F, et al. ABC transporters during epilepsy and mechanisms underlying multidrug resistance in refractory epilepsy. Epilepsia. 2007;48 Suppl 5:140-9. doi: 10.1111/j.15281167.2007.01302.x.

55. Ghosh C, Puvenna V, Gonzalez-Martinez J, et al. Blood-brain barrier P450 enzymes and multidrug transporters in drug resistance: a synergistic role in neurological diseases. Current drug metabolism. 2011;12(8):742-749. doi: 10.2174/138920011798357051.

56. Vazquez SE, D’Giano C, Carpintiero S, et al. Increase 99mTc-SESTAMIBI (MIBI) liver clearance could have identified epileptic pharmacoresistant patients. A preliminary study. Epilepsia. 2004;45:120.

57. Chiosa V, Groppa SA, Ciolac D, et al. Breakdown of thalamo-cortical connectivity precedes spike generation in focal epilepsies. Brain Connect. 2017;7(5):309-320. doi: 10.1089/brain.2017.0487.

58. Chiosa V, Ciolac D, Groppa S, et al. Large-scale network architecture and associated structural cortico-subcortical abnormalities in patients with sleep/awake-related seizures. Sleep. 2019;42(4):zsz006.. doi: 10.1093/ sleep/zsz006.

59. Ciolac D. Reorganization and resilience of brain networks in focal epilepsy. Mold Med J. 2020;63(5):5-8. doi: 10.5281/zenodo.4018890.

60. Juvale IIA, Che Has AT. Possible interplay between the theories of pharmacoresistant epilepsy. Eur J Neurosci. 2021;53(6):1998-2026. doi: 10.1111/ejn.15079.

61. Cardenas-Rodriguez N, Carmona-Aparicio L, Pérez-Lozano DL, et al. Genetic variations associated with pharmacoresistant epilepsy (Review). Mol Med Rep. 2020;21(4):1685-1701. doi: 10.3892/mmr.2020.10999.

62. Chouchi M, Kaabachi W, Klaa H, et al. Relationship between ABCB1 3435TT genotype and antiepileptic drugs resistance in Epilepsy: updated systematic review and meta-analysis. BMC Neurol. 2017;17(1):1-14. doi: 10.1186/s12883-017-0801-x.

63. Fernandez-Marmiesse A, Sánchez-Iglesias S, Darling A, et al. A de novo heterozygous missense BSCL2 variant in 2 siblings with intractable developmental and epileptic encephalopathy. Seizure. 2019;71:161-165. doi: 10.1016/j.seizure.2019.07.019.64. Goto A, Ishii A, Shibata M, et al. Characteristics of KCNQ 2 variants causing either benign neonatal epilepsy or developmental and epileptic encephalopathy. Epilepsia. 2019;60(9):1870-1880. doi: 10.1111/epi.16314.

65. Yang X, Yan Y, Fang Set al. Comparison of oxcarbazepine efficacy and MHD concentrations relative to age and BMI: Associations among ABCB1, ABCC2, UGT2B7, and SCN2A polymorphisms. Medicine (Baltimore). 2019;98(12):e14908. doi: 10.1097/MD.0000000000014908.

66. Esmaeilzadeh H, Farjadian S, Alyasin S, et al. Epidemiology of severe cutaneous adverse drug reaction and its HLA Association among pediatrics. Iran J Pharm Res. 2019;18(1):506-522.

67. Myers CT, Mefford HC. Advancing epilepsy genetics in the genomic era. Genome Med. 2015;7(1):91. doi: 10.1186/s13073-015-0214-7.

68. Waldbaum S, Patel M. Mitochondria, oxidative stress, and temporal lobe epilepsy. Epilepsy Res. 2010;88(1):23-45. doi: 10.1016/j. eplepsyres.2009.09.020.

69. Janmohamed M, Brodie MJ, Kwan P. Pharmacoresistance - Epidemiology, mechanisms, and impact on epilepsy treatment. Neuropharmacology. 2020;168:107790. doi: 10.1016/j.neuropharm.2019.107790.

70. Kobow K, Reid CA, van Vliet EA, et al. Epigenetics explained: a topic "primer" for the epilepsy community by the ILAE Genetics/Epigenetics Task Force. Epileptic Disord. 2020;22(2):127-141. doi: 10.1684/ epd.2020.1143.

71. Hauser RM, Henshall DC, Lubin FD. The epigenetics of epilepsy and its progression. Neuroscientist. 2018;24(2):186-200. doi: 10.1177/ 1073858417705840.

72. Miller-Delaney SF, Bryan K, Das S, et al. Differential DNA methylation profiles of coding and non-coding genes define hippocampal sclerosis in human temporal lobe epilepsy. Brain. 2015;138(Pt 3):616-31. doi: 10.1093/brain/awu373.
73. Morris G, Reschke CR, Henshall DC. Targeting microRNA-134 for seizure control and disease modification in epilepsy. EBioMedicine. 2019;45:646-654. doi: 10.1016/j.ebiom.2019.07.008.

74. Vezzani A, Rench J, Bartfai T, et al. The role of inflammation in epilepsy. Nat Rev Neurol. 2011;7(1):31-40. doi: 10.1038/nrneurol.2010.178.

75. Vezzani A, Viviani B. Neuromodulatory properties of inflammatory cytokines and their impact on neuronal excitability. Neuropharmacology. 2015;96(Pt A):70-82. doi: 10.1016/j.neuropharm.2014.10.027.

76. Holmes M, Flaminio Z, Vardhan M, et al. Cross talk between drugresistant epilepsy and the gut microbiome. Epilepsia. 2020;61(12):26192628. doi: 10.1111/epi.16744.

77. Medel-Matus JS, Shin D, Dorfman E, et al. Facilitation of kindling epileptogenesis by chronic stress may be mediated by intestinal microbiome. Epilepsia Open. 2018;3(2):290-294. doi: 10.1002/epi4.12114.

78. Xie G, Zhou Q, Qiu CZ, et al. Ketogenic diet poses a significant effect on imbalanced gut microbiota in infants with refractory epilepsy. World J Gastroenterol. 2017;23(33):6164. doi: 10.3748/wjg.v23.i33.6164.

79. Zhang Y, Zhou S, Zhou Y, et al. Altered gut microbiome composition in children with refractory epilepsy after ketogenic diet. Epilepsy Res. 2018;145:163-168. doi: 10.1016/j.eplepsyres.2018.06.015.

80. Lindefeldt M, Eng A, Darban H, et al. The ketogenic diet influences taxonomic and functional composition of the gut microbiota in children with severe epilepsy. NPJ Biofilms Microbiomes. 2019;5(1):1-13. doi: 10.1038/s41522-018-0073-2.

81. Gómez-Eguílaz M, Ramón-Trapero JL, Pérez-Martínez L, et al. The beneficial effect of probiotics as a supplementary treatment in drugresistant epilepsy: a pilot study. Benef Microbes. 2018;9(6):875-881. doi: 10.3920/BM2018.0018.

82. Yeom JS, Park JS, Kim YS, et al. Neonatal seizures and white matter injury: Role of rotavirus infection and probiotics. Brain Dev. 2019;41(1):19-28. doi: 10.1016/j.braindev.2018.07.001.

83. Larivière S, Bernasconi A, Bernasconi N, et al. Connectome biomarkers of drug-resistant epilepsy. Epilepsia. 2021;62(1):6-24. doi: 10.1111/ epi.16753.

84. Henshall DC, Hamer HM, Pasterkamp RJ, et al. MicroRNAs in epilepsy: pathophysiology and clinical utility. Lancet Neurol. 2016;15(13):13681376. doi: 10.1016/S1474-4422(16)30246-0.

85. Parras A, de Diego-Garcia L, Alves M, et al. Polyadenylation of mRNA as a novel regulatory mechanism of gene expression in temporal lobe epilepsy. Brain. 2020;143(7):2139-2153. doi: 10.1093/brain/awaa168.

86. Lagarde S, Villeneuve N, Trébuchon A, et al. Anti-tumor necrosis factor alpha therapy (adalimumab) in Rasmussen's encephalitis: an open pilot study. Epilepsia. 2016;57(6):956-966. doi: 10.1111/epi.13387.

87. Woo CW, Chang LJ, Lindquist MA, et al. Building better biomarkers: brain models in translational neuroimaging. Nat Neurosci. 2017;20(3):365-377. doi: 10.1038/nn.4478.

88. French JA, Lawson JA, Yapici Z, et al. Adjunctive everolimus therapy for treatment-resistant focal-onset seizures associated with tuberous sclerosis (EXIST-3): a phase 3, randomised, double-blind, placebocontrolled study. Lancet. 2016;388(10056):2153-2163. doi: 10.1016/ S0140-6736(16)31419-2.

89. Zaccara G, Schmidt D. Antiepileptic drugs in clinical development: differentiate or die? Current pharmaceutical design. 2017;23(37):55935605. doi: 10.2174/1381612823666170809100524.

90. Krauss GL, Klein P, Brandt C, et al. Safety and efficacy of adjunctive cenobamate (YKP3089) in patients with uncontrolled focal seizures: a multicentre, double-blind, randomised, placebo-controlled, doseresponse trial. Lancet Neurol. 2020;19(1):38-48. doi: 10.1016/S14744422(19)30399-0.

91. Polster T. Individualized treatment approaches: Fenfluramine, a novel antiepileptic medication for the treatment of seizures in Dravet syndrome. Epilepsy Behav. 2019;91:99-102. doi: 10.1016/j. yebeh.2018.08.021.

92. Szaflarski JP, Bebin EM, Comi AM, et al. Long-term safety and treatment effects of cannabidiol in children and adults with treatment-resistant epilepsies: expanded access program results. Epilepsia. 2018;59(8):15401548. doi: 10.1111/epi.14477. 
93. Chen JW, Borgelt LM, Blackmer AB. Cannabidiol: a new hope for patients with Dravet or Lennox-Gastaut syndromes. Ann Pharmacother. 2019;53(6):603-611. doi: 10.1177/1060028018822124.

94. Simonato M. Gene therapy for epilepsy. Epilepsy Behav. 2014;38:125-30. doi: 10.1016/j.yebeh.2013.09.013.

95. Riban V, Fitzsimons HL, During MJ. Gene therapy in epilepsy. Epilepsia. 2009;50(1):24-32. doi: 10.1111/j.1528-1167.2008.01743.x.

96. Mollanoori H, Teimourian S. Therapeutic applications of CRISPR/ Cas9 system in gene therapy. Biotechnol Lett. 2018;40(6):907-914. doi: 10.1007/s10529-018-2555-y.

97. Rao G, Mashkouri S, Aum D, et al. Contemplating stem cell therapy for epilepsy-induced neuropsychiatric symptoms. Neuropsychiatr Dis Treat. 2017;13:585-596. doi: 10.2147/NDT.S114786.

98. Kalani A, Tyagi A, Tyagi N. Exosomes: mediators of neurodegeneration, neuroprotection and therapeutics. Mol Neurobiol. 2014;49(1):590-600. doi: 10.1007/s12035-013-8544-1.

99. Janmohamed M, Brodie MJ, Kwan P. Pharmacoresistance - Epidemiology, mechanisms, and impact on epilepsy treatment. Neuropharmacology. 2020;168:107790. doi: 10.1016/j.neuropharm.2019.107790.
100.Trinka E, Brigo F. Neurostimulation in the treatment of refractory and super-refractory status epilepticus. Epilepsy Behav. 2019;101(Pt B):106551. doi: 10.1016/j.yebeh.2019.106551.

101.San-juan D, Morales-Quezada L, Orozco Garduño AJ, et al. Transcranial direct current stimulation in epilepsy. Brain Stimul. 2015;8(3):455464. doi: 10.1016/j.brs.2015.01.001.

102.Fanselow EE. Central mechanisms of cranial nerve stimulation for epilepsy. Surg Neurol Int. 2012;3(Suppl 4):S247-54. doi: 10.4103/21527806.103014.

103. Rocchi L, Ibáñez J, Benussi A, et al. Variability and predictors of response to continuous theta burst stimulation: a TMS-EEG study. Front Neurosci. 2018;12:400. doi: 10.3389/fnins.2018.00400.

104. Ellis TL, Stevens A. Deep brain stimulation for medically refractory epilepsy. Neurosurg Focus. 2008;25(3):E11. doi: 10.3171/FOC/2008/25/9/ E11.

105.Téllez-Zenteno JF, Hernández-Ronquillo L, Buckley S, et al. A validation of the new definition of drug-resistant epilepsy by the International League Against Epilepsy. Epilepsia. 2014;55(6):829-834. doi: 10.1111/ epi.12633.

\section{Authors' ORCID iDs and academic degrees}

Vitalie Chiosa, MD, PhD, Assistant Professor - https://orcid.org/0000-0001-9026-1121

Dumitru Ciolac, MD, Assistant Professor - https://orcid.org/0000-0003-1243-313X

Viorica Chelban, MD, PhD, MSc, MRCP, Assistant Professor - https://orcid.org/0000-0002-5817-6290

Daniela Gasnas, MD, Assistant Professor - https://orcid.org/0000-0003-2696-5444

Anatolie Vataman, MD, Assistant Professor - https://orcid.org/0000-0002-6328-6216

Cristina Munteanu, MD, Assistant Professor - https://orcid.org/0000-0001-9534-2094

Stanislav Groppa, MD, PhD, Professor, Academician - https://orcid.org/0000-0002-2120-2408

\section{Authors' contribution}

VC, DC and SG conceptualized the project and designed the research; VC, DC, VC, DG, AV and CM conducted literature review and drafted the first manuscript. SG revised the manuscript critically. All the authors approved the final version of the manuscript.

\section{Funding}

This study was supported by Nicolae Testemitanu State University of Medicine and Pharmacy and Institute of Emergency Medicine, Chisinau, the Republic of Moldova. This study was also supported by the project entitled "Integrating epileptogenic mechanisms to create a network of multimodal diagnosis and treatment of epilepsy" (20.80009.8007.40) within the State Program (2020-2023). The trial was the authors' initiative. The authors are independent and take responsibility for the integrity of the data and accuracy of the data analysis.

\section{Ethics approval and consent to participate}

No approval was required for this study.

\section{Conflict of Interests}

The authors declare no conflict of interests. 